\title{
Skin autofluorescence is increased in patients with carotid artery stenosis and peripheral artery disease
}

\author{
Marjon J. Noordzij • Joop D. Lefrandt • Erik A. H. Loeffen • \\ Ben R. Saleem • Robbert Meerwaldt $\cdot$ Helen L. Lutgers • \\ Andries J. Smit · Clark J. Zeebregts
}

Received: 9 October 2010/Accepted: 13 January 2011/Published online: 19 February 2011

(C) The Author(s) 2011. This article is published with open access at Springerlink.com

\begin{abstract}
Advanced glycation end products (AGEs) have a pivotal role in atherosclerosis. We evaluated skin autofluorescence (SAF), a non-invasive measurement of tissue AGE accumulation, in patients with carotid artery stenosis with and without coexisting peripheral artery occlusive disease (PAOD). SAF was measured using the AGE Reader ${ }^{\mathrm{TM}}$ in 56 patients with carotid artery stenosis and in 56 age- and sex-matched healthy controls without diabetes, renal dysfunction or known atherosclerotic disease. SAF was higher in patients with carotid artery stenosis compared to the control group: mean 2.81 versus $2.46(P=0.002)$, but especially in the younger age group of 50-60 years old: mean 2.82 versus $1.94(P=0.000)$. Patients with carotid artery stenosis and PAOD proved to have an
\end{abstract}

M. J. Noordzij · J. D. Lefrandt · H. L. Lutgers ·

A. J. Smit

Departments of Internal Medicine, Division of Vascular

Medicine, University Medical Center Groningen,

University of Groningen, Groningen, The Netherlands

E. A. H. Loeffen - B. R. Saleem - C. J. Zeebregts $(\bowtie)$ Department of Surgery, Division of Vascular Surgery, University Medical Center Groningen, University of Groningen, P.O. Box 30.001, 9700 RB Groningen,

The Netherlands

e-mail: czeebregts@hotmail.com

R. Meerwaldt

Department of Surgery, Medisch Spectrum Twente,

Enschede, The Netherlands even higher SAF than patients with carotid artery stenosis only: mean 3.28 versus $2.66(P=0.003)$. Backward linear regression analysis showed that age, smoking, diabetes mellitus, renal function and the presence of PAOD were the determinants of SAF, but carotid artery stenosis was not. SAF is increased in patients with carotid artery stenosis and PAOD. The univariate and multivariate associations of SAF with age, smoking, diabetes, renal insufficiency and PAOD suggest that increased SAF can be seen as an indicator of widespread atherosclerosis.

Keywords Carotid artery stenosis - Peripheral vascular disease - Advanced glycation end products . Skin autofluorescence

\section{Introduction}

Advanced glycation end products (AGEs) accumulate in long lived tissue during lifetime, which is regarded as a process of normal ageing. AGE accumulation results from a combination of hyperglycaemia, hyperlipaemia, oxidative/carbonyl stress and also decreased renal clearance of AGE precursors. Accelerated AGE accumulation is therefore, seen in diabetes mellitus and renal failure and contributes to long term complications and mortality [1-3] AGEs also play a major pathogenetic role in atherosclerosis [4-7]. Cross linking of AGEs with collagen and elastin within the vascular wall contributes to arterial 
stiffness $[1,5,6]$. AGEs also alter the extracellular matrix and promote atheroma formation [6-8]. Furthermore, activation of cell membrane receptors including the receptor for AGE (RAGE) leads to activation of several oxidative and inflammatory pathways $[1,7,8]$. This cascade leads to endothelial dysfunction, vascular inflammation and production of reactive oxygen species [1]. These mechanisms accelerate the formation of atherosclerosis [1]. Finally, AGE accumulation and overexpression of RAGE within the plaque may promote plaque instability [6].

Accumulation of AGEs in tissue can be assessed through illumination of the skin, a technique named skin autofluorescence (SAF), which has previously been validated by simultaneous measurements of SAF and contents of specific AGE assessments in skin biopsies [9-11]. Although the fluorescent characteristics in this method are not specific for fluorescent AGE, multiple validation studies have shown convincingly and consistently that SAF has a strong correlation with specific AGE content in skin biopsies [9-11]. The correlation between SAF with the fluorescent AGE pentosidine is very high: $r=0.87$. Surprisingly, not only fluorescent AGE (pentosidine) but also non fluorescent AGE (N-carboxymethyllysine (CML) and N-carboxyethyl-lysine (CEL)) in the skin biopsies showed great correlation with SAF. Skin AGE content explained the major part of the variance (up to $76 \%$ ) in the SAF signal in a pooled analysis of three validation studies [10]. We earlier reported increased SAF in several groups of patients with increased AGEs formation such as diabetes mellitus [3, 12], decreased clearance of AGEs such as renal failure [13] and overt atherosclerotic disease such as patients with stable coronary artery disease [14]. Earlier studies have already demonstrated an elevated serum level of AGEs in patients with carotid disease; a positive association between intima media thickness (IMT) and serum levels of AGEs were found in population with renal insufficiency starting dialysis [15]. Baumann et al. showed that the AGE $\mathrm{N}$-epsilon-carboxymethyllysine (CML) is present in the subendothelial space of atherosclerotic human carotid artery material of normoglycaemic subjects with a mean age of 50 years [16]. However, the level of SAF as a measurement of increased tissue accumulation of AGEs rather than plasma AGEs level has not yet been studied in patients with carotid artery stenosis. Therefore, the present study evaluates SAF in patients with atherosclerotic carotid artery stenosis with or without coexisting peripheral arterial disease. The possible value of SAF as a risk indicator in this specific cohort of patients is further discussed.

\section{Materials and methods}

Subjects

Between October 2007 and May 200856 consecutive patients with carotid artery stenosis admitted to the outpatient clinic of the Department of Surgery (Division of Vascular Surgery) in our tertiary referral hospital participated in the study after informed consent was obtained. The degree of stenosis and basic morphologic features of the plaque were evaluated by duplex ultrasound. Duplex ultrasound was performed in the clinical setting as part of standard medical care. This was performed by one observer, a specialized sonographer of the department of vascular surgery of our university hospital. Inclusion criteria were the presence of a symptomatic stenosis of 70-99\% (or less in case of ulcerative soft plaques) or an asymptomatic stenosis of $80-99 \%$. There were no exclusion criteria. 56 age- and sexmatched controls were recruited at the outpatient clinic of the Department of Anaesthesiology for preoperative evaluation prior to an elective noncardiovascular related surgical procedure. Control patients were eligible if they did not have a history of diabetes, cardiovascular disease or renal disease. Carotid ultrasound was not performed in the control group.

\section{Study protocol}

In both groups, classical cardiovascular risk factors as well as other factors that are known to influence AGE accumulation were inventoried: body mass index, smoking status, diabetes mellitus, hypertension (defined as a blood pressure of more than 140/90 $\mathrm{mmHg}$ or the use of antihypertensive medication), hypercholesterolemia (depicted by the use of statins), renal function and the presence of coronary artery disease and peripheral artery occlusive disease (PAOD). SAF was measured in carotid artery stenosis and control patients. 
Assessment of skin autofluorescence

SAF was measured with the AGE Reader ${ }^{\text {TM }}$ (DiagnOptics Technologies BV, Groningen, the Netherlands). The AGE Reader ${ }^{\mathrm{TM}}$ is a desk-top device that uses the characteristic fluorescent properties of some AGEs to estimate the level of AGE accumulation in the skin. Technical details of this non-invasive device concerning the optical technique have been described more extensively elsewhere [10]. In short, the AGE Reader illuminates a skin surface of $4 \mathrm{~cm}^{2}$ guarded against surrounding light with an excitation light source with a peak excitation of $370 \mathrm{~nm}$. Emission light (fluorescence in the wavelength of 420-600 nm) and reflected excitation light (with a wavelength of $300-420 \mathrm{~nm}$ ) from the skin is measured with a spectrometer. SAF is calculated as the ratio between the emission light and reflected excitation light, multiplied by 100 and expressed in arbitrary units.

In the current series of experiments, the right forearm was positioned on top of the device. The right forearm is the standard measuring site for SAF as it is the most practical site and autofluorescence is believed to be uniformly distributed throughout the body. A series of three consecutive measurements was carried out, which took less than a minute time. The mean SAF of three consecutive measurements was calculated and used in the analyses. An earlier validation study showed an intra individual Altman error percentage of $5.03 \%$ with SAF measurements taken over 1 single day and an Altman error percentage of $5.87 \%$ over seasonal variation [10]. Between subjects, SAF has a standard deviation of approximately 0.5 AU [17]. SAF shows a linear increase with age of 0.023 AU per year for subjects up to 70 years [17]. Gender had no influence on SAF in non-smokers. In smokers, SAF was 0.2 AU higher in females [17]. The SAF measurement with the AGE reader is independent of skin colour when reflectance values are above $12 \%$ as was the case in all of the study participants [10]. Measurements were performed in fasting state as previous studies showed a postprandial rise up to $12 \%[18,19]$.

\section{Statistical analysis}

Power analysis was based on the reference values for SAF provided by Koetsier et al. [17]. Using a mean $\mathrm{SAF}$ of $2.5 \mathrm{AU}$ with a SD of $0.55 \mathrm{AU}, 56$ patients and
56 controls were needed to detect a difference of at least $0.3 \mathrm{AU}(=12 \%)$ with a power of $80 \%$ and a $P$-value $<0.05$. Data were prospectively gathered in a database (SPSS 15-0, SPSS Inc, Chicago, Illinois, USA). Distribution of variables was tested by the Kolmorgorov-Smirnov test. All parameters showed a normal distribution. Descriptive statistics are therefore, presented as mean with standard deviation or as number of patients. For comparison between continuous variables the $t$-test was used. For categorical variables the Fisher exact test was used. Correlations between variables were analysed by Pearson's correlation. Subsequently, backward linear regression analysis was performed to determine the parameters that independently influenced SAF. A $P$-value less than 0.05 was regarded as statistically significant.

\section{Results}

Subject characteristics

Patient characteristics are summarized in Table 1. The mean age of both groups was approximately 69 years. All patients were Caucasian and thirtyseven patients $(66 \%)$ were male in both groups. As expected, the presence of traditional cardiovascular risk factors as well as established coronary artery or peripheral artery occlusive disease (PAOD) was significantly higher in the patients with carotid artery stenosis. Twenty five patients with carotid artery stenosis were current smokers compared to 9 in the control group. Mean BMI was not different between the groups, both approximately $27.5 \mathrm{~kg} / \mathrm{m}^{2}$. Twelve patients $(21 \%)$ had diabetes mellitus and three of them used insulin therapy. Mean blood pressure was $153 / 80 \mathrm{mmHg}$ in the patients with carotid artery disease and $156 / 84 \mathrm{mmHg}$ in the control group. Diastolic blood pressure was significantly higher in the control group. Nearly all patients with carotid artery stenosis had hypertension $(n=54)$ and nearly half of them used more than two antihypertensive agents. Significantly fewer subjects in the control group had hypertension ( $n=43$ ) but only 18 of these hypertensive controls received antihypertensive medication. Statins were used by 49 patients with carotid artery stenosis $(87.5 \%)$, while only one subject in the control group was on statin therapy. All patients with carotid artery stenosis used either antiplatelet therapy 
Table 1 Baseline characteristics presented as mean (standard deviation), median (interquartile range) or as number of patients $(\%)$

\begin{tabular}{lllc}
\hline Characteristic & Carotid artery stenosis & Control subjects & $P$ \\
\hline $\mathrm{N}$ & 56 & 56 & \\
Age (years) & $69.0(7.8)$ & $68.9(7.9)$ & 0.96 \\
Male sex & $37(66 \%)$ & $37(66 \%)$ & 1.0 \\
Current smoker & $25(45 \%)$ & $9(16 \%)$ & 0.002 \\
BMI (kg/m2) & $27.2(4.9)$ & $27.8(3.9)$ & 0.49 \\
Diabetes mellitus & $12(21 \%)$ & 0 & 0.001 \\
Diabetes mellitus requiring insulin & $3(5 \%)$ & 0 & 0.24 \\
Systolic blood pressure & $153(22)$ & $156(22)$ & 0.47 \\
Diastolic blood pressure & $80(74-89)$ & $84(80-89)$ & 0.016 \\
Hypertension (>140/90 mmHg/med) & $54(96 \%)$ & $43(77 \%)$ & 0.004 \\
Antihypertensive medication & $52(93 \%)$ & $18(32 \%)$ & $<0.001$ \\
$>2$ antihypertensive agents & $22(39 \%)$ & $2(4 \%)$ & $<0.001$ \\
Statin therapy & $49(88 \%)$ & $1(2 \%)$ & $<0.001$ \\
Serum creatinine & $90.1(34.5)$ & $95.6(15.7)$ & 0.28 \\
Renal clearance (MDRD) & $84(37)$ & $73(16.7)$ & 0.050 \\
Anti platelet therapy & $50(89 \%)$ & 0 & $<0.001$ \\
Coronary artery disease & $17(30 \%)$ & 0 & $<0.001$ \\
PAOD & $14(25 \%)$ & 0 & $<0.001$ \\
Coronary artery disease and/or PAOD & $26(46 \%)$ & 0 & $<0.001$ \\
\hline & & & \\
\hline
\end{tabular}

$(n=50)$ or an oral vitamin $\mathrm{K}$ inhibitor $(n=6)$. Mean serum creatinin was 90 in the patients with carotid artery stenosis and $96 \mathrm{umol} / \mathrm{l}$ in the control group. Nearly half of the patients with carotid artery stenosis also had either coronary artery disease or peripheral artery occlusive disease $(n=26)$. Of the 56 patients with carotid artery disease, 14 had coexisting PAOD and 17 coexisting coronary artery disease. As a consequence of the exclusion criteria, none of the control group patients had atherosclerotic manifestations.

\section{Skin autofluorescence}

Overall, SAF was significantly higher in the patients with carotid artery stenosis (mean SAF 2.81) compared to the control group (mean SAF 2.46) (Table 2). After stratification for age, the difference in SAF was primarily observed in the age category of 50-60 years: a mean SAF of 2.82 in the carotid artery stenosis patients versus a mean SAF of 1.94 in the controls. (Table 2). After exclusion of patients with diabetes mellitus the differences in SAF remained significant in the total group with carotid artery stenosis as well as in the age subgroup of 50-60 years old. Figure 1 shows that the control group exhibits the natural increase of SAF with advancing age ( $r=0.52, P<0.001)$. In the patient group with carotid artery stenosis, the relationship between SAF and age has disappeared as younger patients already have a high SAF $(r=0.094, P=0.49)$.

Within the carotid artery stenosis group, the degree of stenosis did not influence SAF $(P=0.71)$. Also, no difference in SAF was found between the asymptomatic and symptomatic patients with carotid artery stenosis $(P=0.21)$.

PAOD proved to be strongly associated with a high SAF. Patients with PAOD had a higher SAF than patients without peripheral arterial occlusive disease. When comparing SAF of patients with carotid artery stenosis and PAOD to SAF in the control group, SAF was $3.28(0.66)$ versus $2.46(0.57)$ with a $P$ value of 0.000 (Table 2). Even within the group of carotid artery stenosis, SAF was significantly higher when there was coexisting PAOD. Subjects with both carotid artery stenosis and PAOD had a SAF of $3.28(0.66)$ versus $2.66(0.53)$ for subjects with only carotid artery stenosis without coexisting PAOD. When excluding patients with PAOD, the difference in SAF between the patients with carotid artery stenosis and controls was limited and even lost statistical significance: SAF was 2.66 
Table 2 Skin

autofluorescence in patients with carotid artery stenosis compared with healthy controls

Analysis of different age groups. Peripheral artery occlusive disease and coronary artery disease are denoted by, respectively PAOD and CAD. Statistical significance is notated by*

\begin{tabular}{llrlrl}
\hline Skin autofluorescence & $\begin{array}{l}\text { Carotid artery } \\
\text { stenosis }\end{array}$ & $N$ & Control subjects & $N$ & $P$-value \\
\hline Total group & $2.81(0.62)$ & 56 & $2.46(0.57)$ & 56 & $0.002^{*}$ \\
Total group without diabetes & $2.75(0.64)$ & 44 & $2.46(0.57)$ & 56 & $0.016^{*}$ \\
Total group with PAOD & $3.28(0.66)$ & 14 & $2.46(0.57)$ & 56 & $0.000^{*}$ \\
Total group without PAOD & $2.66(0.53)$ & 42 & $2.46(0.57)$ & 56 & 0.084 \\
Total group with CAD & $2.82(0.46)$ & 17 & $2.46(0.57)$ & 56 & $0.021^{*}$ \\
Total group without CAD & $2.81(0.69)$ & 39 & $2.46(0.57)$ & 56 & $0.008^{*}$ \\
50-60 years & $2.82(0.54)$ & 8 & $1.94(0.30)$ & 10 & $0.000^{*}$ \\
50-60 years without diabetes & $2.79(0.37)$ & 5 & $1.94(0.30)$ & 10 & $0.000^{*}$ \\
60-70 years & $2.73(0.58)$ & 23 & $2.40(0.54)$ & 21 & 0.06 \\
$70-80$ years & $2.90(0.71)$ & 21 & $2.73(0.55)$ & 24 & 0.37 \\
$>80$ years & $2.83(0.74)$ & 4 & $2.19(n=1)$ & 1 & 0.49 \\
\hline
\end{tabular}

higher SAF. Subjects with carotid artery stenosis and also coronary artery disease had a SAF of $2.82(0.69)$ while this was $2.81(0.46)$ for patients with carotid artery disease without coexisting coronary disease.

Correlations and univariate analysis

Univariate analysis and correlations were performed in the combined total group to determine the relationship of different parameters with SAF. A significant correlation of SAF was found with age $(r=0.29, P<0.01)$ and with renal function $(r=$ $0.24, P=0.01)$. A significantly higher SAF was found in current smokers (mean 3.01 versus mean $2.47, P<0.001$ ), diabetes mellitus (mean 3.03 versus 2.59, $P=0.02$ ), those using statins (mean 2.87 versus $2.44, P<0.001$ ), those with hypertension (mean 2.73 versus $2.48, P=0.04$ ) and those with coexistence of PAOD (mean 3.28 versus 2.46, $P<0.001)$. Gender, BMI, hypertension and coronary artery disease had no significant influence in univariate analysis.

\section{Backward linear regression analysis}

Backward linear regression analysis was performed in the combined total group to determine the parameters that independently contributed to SAF. The results are shown in Table 3. Age, smoking, diabetes mellitus, renal function and presence of PAOD were the significant determinants of SAF. In contrast to the results of univariate analysis the presence of carotid artery disease did not contribute significantly with a $\beta$ 
Table 3 Results of backward linear regression analysis

\begin{tabular}{lll}
\hline Included parameter & Beta & $P$-value \\
\hline Age & 0.24 & 0.02 \\
Current smoker & 0.36 & 0.00 \\
Diabetes mellitus & 0.21 & 0.01 \\
EGFR (MDRD) & 0.20 & 0.05 \\
Peripheral artery occlusive disease & 0.29 & 0.00 \\
\hline
\end{tabular}

The remaining significant predictors of skin autofluorescence are shown. Carotid artery disease, coronary artery disease, sex, BMI, hypertension and use of statin medication did not significantly influenced skin autofluorescence. Carotid artery disease and coronary artery disease both did not contribute significantly with, respectively a $\beta$ of $0.074(P=0.83)$ and $0.038(P=0.48)$

of $0.074(P=0.83)$. Coronary artery disease also did not contribute with a $\beta$ of $0.038(P=0.48)$.

\section{Discussion}

In the current study we showed that SAF is significantly elevated in patients with carotid artery stenosis and PAOD compared to controls. Two findings were especially remarkable.

First, PAOD proved to be an important determinant of SAF. High SAF values were especially found in the group with carotid artery disease and coexisting PAOD. Even within the group of carotid artery disease, SAF was significantly higher when there was coexisting PAOD with a SAF of 3.36 versus 2.64 $(P=0.003)$. This could not be explained by differences in baseline characteristics between the patients with carotid artery stenosis with or without PAOD. Also in linear regression analysis, PAOD proved to be a strong determinant of SAF, even more so then diabetes. This finding is interesting and warrants further research. It suggests that SAF should primarily be seen as an indicator of widespread atherosclerotic disease. Currently, a study analysing SAF in patients with primary peripheral artery disease has been initiated.

The second remarkable finding was that SAF was specifically elevated for patients in the age group 50-60 years. Furthermore, the relation between age and SAF, as normally seen, was present in the control group but absent in the patients with carotid artery stenosis.
The marked elevation of SAF levels in carotid artery stenosis was demonstrated in non-diabetic patients as well as in diabetics. A high SAF in diabetes mellitus may therefore, be regarded as an indicator of widespread atherosclerotic disease and not only a manifestation of diabetes mellitus per se. Mulder et al. previously reported similar findings in a cohort with stable coronary artery disease [14]. SAF was significantly increased in stable coronary artery disease compared with controls, irrespective of diabetes, current smoking and renal function. Earlier studies in patients with diabetes mellitus also showed that SAF is manifestly increased in these patients. The level of AGE accumulation correlated with the duration and the grade of complications of diabetes $[2,3,20]$.

Univariate and multivariate analysis of associations with SAF in the present study supports this interpretation in our study group of patients with carotid artery stenosis. SAF was univariately associated with age, smoking, diabetes mellitus, renal dysfunction, dyslipidaemia and the presence of carotid artery disease and peripheral arterial occlusive disease which is in concordance with earlier studies [2, 3, 10, 13] Multivariately, SAF was determined by age, smoking, diabetes mellitus, renal dysfunction and peripheral arterial occlusive disease. Therefore, again, increased SAF may rather be regarded as an indicator of widespread atherosclerotic disease than as a specific identifier of carotid atherosclerotic disease.

This study however, has several limitations. At baseline, despite matching for sex and age in the control group, there were differences between the patient and control groups including presence of diabetes, smoking behaviour, hypertension, use of antihypertensive medication and use of statins. This may be considered a source of confounding. However, it does represent the expected increased presence of risk factors for cardiovascular disease in patients with carotid artery stenosis compared to healthy people.

In the control group asymptomatic atherosclerosis may have existed as no carotid ultrasound or other vascular tests were performed in the control group. If asymptomatic atherosclerosis was present in the control group this would result in an underestimation of the difference between the control group and patient group. The differences we found between the 
control group and the group with atherosclerotic carotid stenosis and peripheral artery disease therefore, would be even greater in a better selected control group.

Our study is also limited by the small number of patients. The fact that backward linear regression did not show carotid artery disease or coronary artery disease to be independent determinants of SAF may therefore, be caused by the lack of power. Mulder et al. has earlier shown that SAF indeed is elevated in stable coronary disease [14]. A large study evaluating the relationship between SAF en intima media thickness is currently being executed to further clarify this issue. What may be the use of the present results? The correlation of SAF with traditional cardiovascular risk factors, the presence of diabetes, renal insufficiency and peripheral arterial occlusive disease could indicate that SAF may be an indicator of high cardiovascular risk patients. Moreover, since SAF represents end organ damage it may predict morbidity and mortality more than the classical risk factors for atherosclerosis separately. For patients with type 2 diabetes this has already been established since SAF added prognostic information to the UKPDS risk calculator in predicting mortality [21]. Strong predictive results of SAF for cardiovascular mortality were also seen in patients with renal failure [22]. The same may be true for patients with carotid artery stenosis and peripheral artery disease. Prospective follow-up studies are necessary to elucidate this. Moreover, spectroscopy techniques show promising results for imaging vulnerable plaques and the near future will tell whether they really shine light on unstable cardiovascular disease.

In conclusion, skin autofluorescence is increased in patients with carotid artery stenosis and PAOD compared to healthy controls. SAF is especially elevated in the age group of 50-60 years, suggesting an increased accumulation of tissue AGEs in these patients. The univariate and multivariate associations of SAF with age, smoking, diabetes mellitus, presence of renal insufficiency and peripheral arterial occlusive disease suggest that increased SAF should primarily be seen as an indicator of widespread atherosclerotic disease. These associations further underscore the important role of AGEs in the pathophysiology of atherosclerotic disease. SAF might therefore, be an indicator of an overall high burden of widespread atherosclerosis as a consequence of AGE accumulation. Future research should investigate the use of SAF as a superior predictor of cardiovascular events in this population.

Conflict of interest A.J. Smit is founder of DiagnOptics B.V., Groningen, The Netherlands, manufacturer of the AGE Reader $^{\mathrm{TM}}$, which has been used to perform skin autofluorescence measurements as reported in this manuscript.

Open Access This article is distributed under the terms of the Creative Commons Attribution Noncommercial License which permits any noncommercial use, distribution, and reproduction in any medium, provided the original author(s) and source are credited.

\section{References}

1. Goldin A, Beckman JA, Schmidt AM, Creager MA (2006) Advanced glycation end products: sparking the development of diabetic vascular injury. Circulation 114:597-605

2. Monnier VM, Bautista O, Kenny D, Sell DR, Fogarty J, Dahms W, Cleary PA, Lachin J, Genuth S (1999) Skin collagen glycation, glycoxidation, and crosslinking are lower in subjects with long-term intensive versus conventional therapy of type 1 diabetes: relevance of glycated collagen products versus HbA1c as markers of diabetic complications. DCCT Skin Collagen Ancillary Study Group. Diabetes Control and Complications Trial. Diabetes 48:870-880

3. Lutgers HL, Graaff R, Links TP, Ubink-Veltmaat LJ, Bilo HJ, Gans RO, Smit AJ (2006) Skin autofluorescence as a noninvasive marker of vascular damage in patients with type 2 diabetes. Diabetes Care 29:2654-2659

4. Brownlee M (1995) Advanced protein glycosylation in diabetes and aging. Annu Rev Med 46:223-234

5. Yoshida N, Okumura K, Aso Y (2005) High serum pentosidine concentrations are associated with increased arterial stiffness and thickness in patients with type 2 diabetes. Metabolism 54:345-350

6. Meerwaldt R, van der Vaart MG, van Dam GM, Tio RA, Hillebrands JL, Smit AJ, Zeebregts CJ (2008) Clinical relevance of advanced glycation endproducts for vascular surgery. Eur J Vasc Endovasc Surg 36:125-131

7. Basta G (2008) Receptor for advanced glycation endproducts and atherosclerosis: from basic mechanisms to clinical implications. Atherosclerosis 196:9-21

8. Baynes JW, Thorpe SR (2000) Glycoxidation and lipoxidation in atherogenesis. Free Radic Biol Med 28:1708-1716

9. Meerwaldt R, Links T, Graaff R, Thorpe SR, Baynes JW, Hartog J, Gans R, Smit A (2005) Simple noninvasive measurement of skin autofluorescence. Ann NY Acad Sci 1043:290-298

10. Meerwaldt R, Graaff R, Oomen PH, Links TP, Jager JJ, Alderson NL, Thorpe SR, Baynes JW, Gans RO, Smit AJ (2004) Simple non-invasive assessment of advanced glycation endproduct accumulation. Diabetologia 47:1324-1330

11. Mulder DJ, Water TV, Lutgers HL, Graaff R, Gans RO, Zijlstra F, Smit AJ (2006) Skin autofluorescence, a novel marker for glycemic and oxidative stress-derived advanced 
glycation endproducts: an overview of current clinical studies, evidence, and limitations. Diabetes Technol Ther 8:523-535

12. Meerwaldt R, Lutgers HL, Links TP, Graaff R, Baynes JW, Gans RO, Smit AJ (2007) Skin autofluorescence is a strong predictor of cardiac mortality in diabetes. Diabetes Care 30:107-112

13. Hartog JW, de Vries AP, Lutgers HL, Meerwaldt R, Huisman RM, van Son WJ, de Jong PE, Smit AJ (2005) Accumulation of advanced glycation end products, measured as skin autofluorescence, in renal disease. Ann NY Acad Sci 1043:299-307

14. Mulder DJ, van Haelst PL, Gross S, de Leeuw K, Bijzet J, Graaff R, Gans RO, Zijlstra F, Smit AJ (2008) Skin autofluorescence is elevated in patients with stable coronary artery disease and is associated with serum levels of neopterin and the soluble receptor for advanced glycation end products. Atherosclerosis 197:217-223

15. Suliman ME, Stenvinkel P, Jogestrand T, Maruyama Y, Qureshi AR, Barany P, Heimburger O, Lindholm B (2006) Plasma pentosidine and total homocysteine levels in relation to change in common carotid intima-media area in the first year of dialysis therapy. Clin Nephrol 66:418-425

16. Baumann M, Richart T, Sollinger D, Pelisek J, Roos M, Kouznetsova T, Eckstein HH, Heemann U, Staessen J (2009) Association between carotid diameter and the advanced glycation endproduct Nepsilon-Carboxymethyllysine (CML). Cardiovasc Diabetol 8:45
17. Koetsier M, Lutgers HL, de Jonge C, Links TP, Smit AJ, Graaff R (2010) Reference values of skin autofluorescence. Diabetes Technol Ther 12:399-403

18. Stirban A, Nandrean S, Negrean M, Koschinsky T, Tschoepe D (2008) Skin autofluorescence increases postprandially in human subjects. Diabetes Technol Ther 10:200-205

19. Noordzij MJ, Lefrandt JD, Graaff R, Smit AJ (2010) Skin autofluorescence and glycemic variability. Diabetes Technol Ther 12:581-585

20. Genuth S, Sun W, Cleary P, Sell DR, Dahms W, Malone J, Sivitz W, Monnier VM (2005) Glycation and carboxymethyllysine levels in skin collagen predict the risk of future 10 -years progression of diabetic retinopathy and nephropathy in the diabetes control and complications trial and epidemiology of diabetes interventions and complications participants with type 1 diabetes. Diabetes 54:3103-3111

21. Lutgers HL, Gerrits EG, Graaff R, Links TP, Sluiter WJ, Gans RO, Bilo HJ, Smit AJ (2009) Skin autofluorescence provides additional information to the UK Prospective Diabetes Study (UKPDS) risk score for the estimation of cardiovascular prognosis in type 2 diabetes mellitus. Diabetologia 52:789-797

22. Meerwaldt R, Hartog JW, Graaff R, Huisman RJ, Links TP, den Hollander NC, Thorpe SR, Baynes JW, Navis G, Gans RO, Smit AJ (2005) Skin autofluorescence, a measure of cumulative metabolic stress and advanced glycation end products, predicts mortality in hemodialysis patients. J Am Soc Nephrol 16:3687-3693 